\title{
Commentary
}

\section{CT/MRI brain scan findings: Significant or not?}

\section{Cesar M. Limjoco* \\ *VP, Clinical Services at DCBA, Indianapolis, Indiana, USA}

\section{Commentary}

Early radiographic signs of increased intracranial pressure such as mild vasogenic edema, slight midline shift or early uncal herniation may seem ominous to the lay person. But are they truly significant and impact on the patient's health? Singularly, any of the above findings may not be clinically significant. But together, they may be ominous and signal impending death. One has to always correlate any abnormal findings to the patient's signs and symptoms as well as the treatment modalities that are provided the patient. The above CT/MRI findings, when significant, may reflect an increased intracranial pressure from brain tumors, hematomas/hemorrhages, head injury, hydrocephalus, encephalitis/meningitis, strokes and seizures. The increased intracranial pressure can damage the brain or spinal cord by pressing on important structures and obstruction of blood flow into the brain.

Increased intracranial pressure can be caused by increase in volume in the skull as may occur in cerebral contusion or laceration or intracranial bleed, rapidly enlarging mass or rapid accumulation of cerebrospinal fluid in the ventricles or it can be caused by brain swelling overall as in infections (encephalitis, encephalomyelitis, brain abscess) or can be vasogenic (the blood brain barrier is disrupted) as may occur around cerebral tumors or around any of the above conditions.
Symptoms of increased intracranial pressure include: changes in mental status, behavioral changes, headaches, neurological symptoms (weakness, numbness, eye movement problems, double vision, seizures) and vomiting. Fundoscopic finding of papilledema occurs with several days of increased intracranial pressure. Increased pressure can result in reversible or permanent neurological problems, seizures, strokes/ hemorrhage and death. Sudden increased intracranial pressure is life threatening and needs prompt treatment.

Management involves mechanical and pharmacologic therapies. Head elevation is key to increase venous drainage from the brain. Hyperventilation induces hypocarbia and causes cerebral arterial vasoconstriction and reduces the intracranial pressure. Diuretics like Lasix and Mannitol draw the fluid out of the brain. Glucocorticoids like Decadron and Solumedrol reduce vasogenic edema and can cause marked improvement of symptoms.

Draining the CSF fluid via a ventriculostomy or performing a decompressive craniotomy effectively reduces the pressure in the brain, regardless of cause. Neurosurgical removal of cause of the increased intracranial pressure will be the best means of controlling the intracranial pressure in the long run.
Copyright: (C2016 Limjoco CM. This is an open-access article distributed under the terms of the Creative Commons Attribution License, which permits unrestricted use, distribution, and reproduction in any medium, provided the original author and source are credited.
Correspondence to: Cesar M. Limjoco, VP, Clinical Services at DCBA, Indianapolis, Indiana, USA E-mail: dr_cesar_limjoco@me.com

Received: May 02, 2016; Accepted: June 21, 2016; Published: June 24, 2016 